Unordentlich, langhaarig und mit der Matratze auf dem Boden : Zur Protestsemiotik von Körper und Raum in den 1968er-Jahren

\author{
Linke, Angelika
}

DOI: https://doi.org/10.1515/9783110254723.201

Posted at the Zurich Open Repository and Archive, University of Zurich ZORA URL: https://doi.org/10.5167/uzh-64847

Book Section

Published Version

Originally published at:

Linke, Angelika (2012). Unordentlich, langhaarig und mit der Matratze auf dem Boden : Zur Protestsemiotik von Körper und Raum in den 1968er-Jahren. In: Kämper, Heidrun; Scharloth, Joachim; Wengeler, Martin. 1968: Eine sprachwissenschaftliche Zwischenbilanz. Berlin, Germany: de Gruyter, 201-226.

DOI: https://doi.org/10.1515/9783110254723.201 
Angelika Linke

\section{Unordentlich, langhaarig und mit der Matratze auf dem Boden \\ Zur Protestsemiotik von Körper und Raum in den 1968er Jahren}

\section{Körperzeichen gesellschaftlichen Wandels}

Perioden verdichteten politischen bzw. soziokulturellen Wandels sind häufig verschränkt mit auffälligen Veränderungen im körperlichen und körperkommunikativen Habitus derjenigen sozialen Formationen, die diesen Wandel tragen oder zumindest direkt in ihn eingebunden sind. Solche Veränderungen lassen sich einerseits von außen, aus der Beobachterperspektive konstatieren, wobei eine gewisse historische Distanz zur Schärfung des Blicks beitragen kann. Andererseits werden solche Veränderungen aber auch zeitgenössisch wahrgenommen und kommentiert. Die Erscheinung des Leibes, in der signifikante Merkmale von Kleidung, Haartracht und Schmuck mit den Musterhaftigkeiten von Haltung, Bewegung, Gestik und Mimik zu einer Gesamtheit verschmelzen, bildet - in der Face-toFace-Kommunikation - ein nicht übersehbares Körper-Zeichen, das zudem immer schon in den Bezug auf andere Körper eingebunden ist und diese notwendig affiziert. Wir treten vor und oft auch jenseits jeder verbalen Kommunikation in ein körperliches Verhältnis zur unseren Interaktionspartnern ein, in eine gegenseitige optische Wahrnehmung ebenso wie in eine konfigurative Positionierung, d.h. in eine räumliche Bezugnahme der Leiber. Beides muss nicht bewusst sein, sondern erfolgt im Normalfall auf einer durch Automatismen geprägten, quasi vorbewussten Ebene des Körperhandelns. Entsprechend gilt beides, die optische Wahrnehmung wie die konfigurative Positionierung, auch für solche Begegnungen, die zufällig und für die daran Beteiligten unwichtig sind und deshalb in erster Linie einen spontan-ausweichenden Charakter haben, wie etwa die Begegnung mit uns gleichgültigen Mitmenschen auf Trottoirs und Treppen. Zudem sind Körper nicht nur auf andere Körper, sondern auch auf den umgebenden privaten oder öffentlichen Raum sowie dessen ,Möblierung' bezogen. Und umgekehrt sind beide, privater wie öffentlicher Raum, nicht zuletzt über ihre körperliche Nutzung durch Einzelne wie durch Gruppen in ihrer Ausdehnung definiert und in ihrer Funktion bestimmt - Raum als sozialer 
Raum kann unter semiotischer Perspektive als Extension wie als Produkt des Körpers betrachtet werden. ${ }^{1}$

In all den bisher angesprochenen Zusammenhängen kommt die Zeichenhaftigkeit des Körpers auf individueller wie auf kollektiver Ebene zum Tragen. Sie erzeugt sowohl individuelle wie kollektive Identität und ihr kommt in der Selbstdefinition und Selbstverständigung sozialer Formationen wie auch in der Abgrenzung gegenüber sozial, kulturell oder historisch , anderen ' Gruppierungen eine eigenständige Funktion zu. ${ }^{2}$ Es ist diese Funktion, die ich in den folgenden Überlegungen ins Zentrum stellen und im Kontext der soziokulturellen Umwälzungen der 1968er Jahre genauer fassen möchte. Zuvor werde ich allerdings eine weiter zurückliegende Epoche in den Blick nehmen und, wenn auch nur knapp und skizzenhaft, auf körpersemiotische Veränderungen im 18. Jahrhundert eingehen. Mit diesem historischen Umweg möchte ich einerseits deutlich machen, dass sich Verschränkungen leibräumlicher Zeichenhaftigkeit mit soziokulturellem Wandel grundsätzlich auch in anderen historischen Epochen beobachten lassen (und damit ein durchgängiges Moment der Kultur- wie der Sozialgeschichte bilden), andererseits möchte ich darauf verweisen, dass eine weiterführende Deutung solcher Verschränkungen stark an der jeweiligen historischen Situation orientiert, d.h. kontextualisiert sein muss (und Einszu-eins-Deutungen etwa im Sinne eines „Vokabulars“ von Körperzeichen über historische Zeiten hinweg gerade nicht möglich sind). Der kollektivstilistische Wert eines körperlichen Habitus definiert sich immer sowohl paradigmatisch, d.h. im Vergleich unterschiedlicher Sozialformationen und kultureller Gruppierungen, als auch syntagmatisch, d.h. durch seine Bezüge zum historischen Vorher und Nachher.

1 Ein solches Verständnis von Raum nicht nur als immer schon gegebene Voraussetzung und Kontext, sondern ebenso als Effekt menschlicher Präsenz, menschlichen Handelns und einer spezifischen „Sinnordnung“ prägt neuere, kulturanalytisch orientierte Forschungsansätze in unterschiedlichen Disziplinen. Grundlegend hierfür sind Überlegungen Ernst Cassirers, der u.a. festhält, dass „,[der] Raum [...] nicht eine schlechthin gegebene, ein für allemal feststehende Struktur [besitzt]; sondern er gewinnt diese Struktur erst kraft des allgemeinen Sinnzusammenhangs, innerhalb dessen sein Aufbau sich vollzieht“ (Cassirer 1985, S. 102, hier zitiert nach Wirth 2008, S. 16).

2 Rolf Sachsse betont in seiner Einleitung zu Michael Ruetz’ Bildband zu 1968 denn auch, dass das Festhalten von allem, „was unter dem Begriff Körpersprache subsumiert wird“, einschließlich Frisur, Schminke und Accessoires, „, neben der bildhaften Verdinglichung [...] die wichtigste Aufgabe der Photographie im Bilden kollektiver Gedächtnisse [ist].“ (Sachsse 1997, S. 18, Hervorheb. im Original) 
2. „Alte Teutsche“ vs. ,jetzige Teutsche“: Vom 17. ins 18. Jahrhundert

Ein erstes historisches Beispiel bietet die Darstellung in Abb. 1. Es handelt sich um das Frontispiz der wohl bekanntesten und einflussreichsten Umgangslehre des frühen 18. Jahrhunderts, der 1727 erschienenen „Einleitung in die Ceremoniel=Wissenschaft der Privatpersonen" von Julius Bernhard von Rohr.
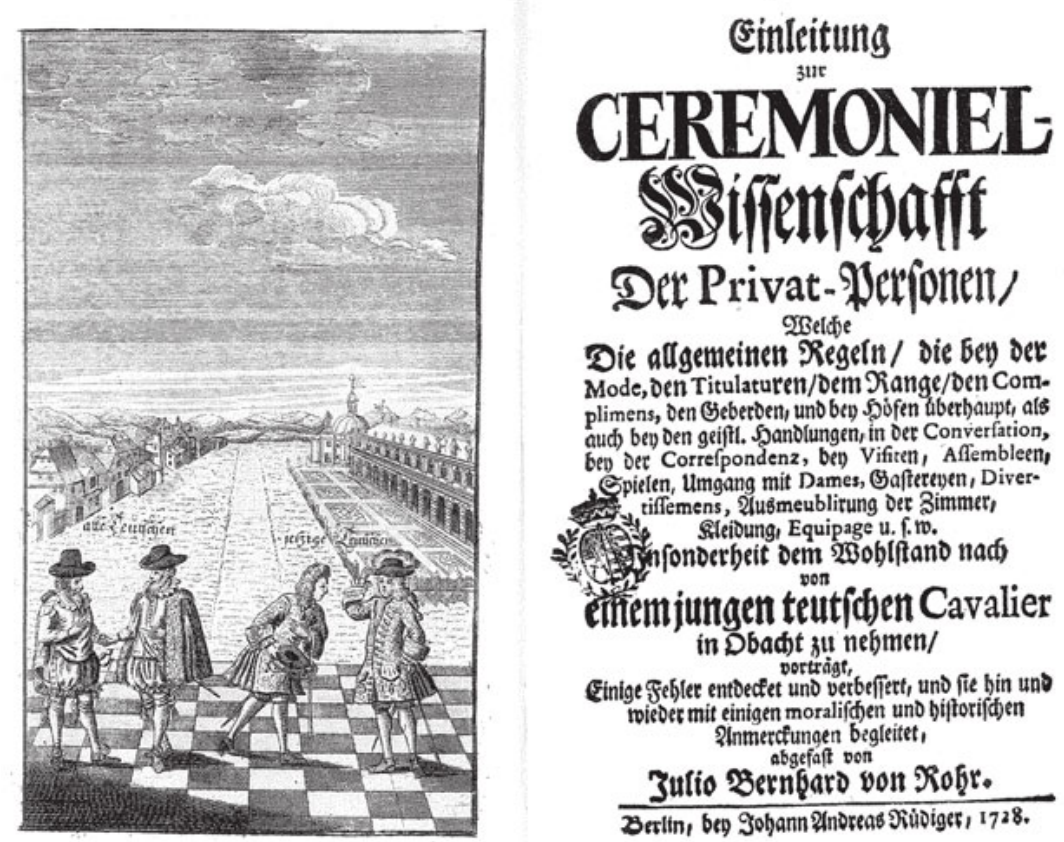

Die allgemeinen Regetn / Die bey Der

Mode, Den Titulaturen/Dem $\Re$ ange/Den Complimens, Den ङseberoen, uno bey Syojfen aberbaupt, als oud beis den geift. Sandlungen, in oer Converfation, bev Der Correfpondenz, ben Vifiten, Aftembleen, Spiefen, Utmgang mit Dames, Bafteteven, Divertiffemens, 2lusmeublitung oer Simmer, Siteioung, Equipage u. .. .w.

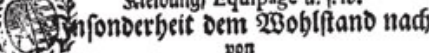
êtîmjungen teutjochen Cavalier in Dbadt) 3 ut nelgmen/

Einige Feblet entoectet und verbeffert, und fie bin uns wieder mit einigen moralif́den uno biftorif̧ben

2inmercfungen begleitet, Zutio ३ernģaro von Kog̨r.

Berlin, ber Johann 2noreas Siüdiges, 1728.

Abb. 1: Einleitung zur Ceremoniel-Wissenschaft Der Privat-Personen. Julius Bernhard von Rohr. Berlin bey Johann Andreas Ruediger, 1728. Frontispiz

Im vorliegenden Kontext interessiert in erster Linie die Behauptung, die das Frontispiz in bildlicher wie sprachlicher Form vornimmt und die gleichzeitig als Legitimation (und Verkaufsargument) für das Buch selbst fungiert: die Behauptung eines historischen Wandels, der im Kontrast von „alten“ und ,jetzigen“ Deutschen sowohl visuell als auch verbal auf einen plakativen Nenner gebracht wird. Die Hauptelemente dieses Wandels, welche durch die Darstellung herausgehoben werden, sind erstens die Kleidung 
und äußere Erscheinung der Figuren, zweitens deren Körperauftritt ${ }^{3}$ und drittens die Szenerie, vor welcher die Figuren präsentiert werden.

Mit Blick auf Kleidung und Äußeres sind v.a. die unterschiedliche Form der Hüte, das Gegenüber von Bart auf der älteren gegenüber der Perücke auf der ,jetzigen“ Seite sowie eine insgesamt steifere, den Faltenwurf betonende und den Unterkörper stärker ausstellende Bekleidung bei den ,alten Teutschen" gegenüber einer schwingenderen, die Linien des Oberkörpers betonende und insgesamt „schnittigeren“ Kleiderlinie bei den ,jetzigen Teutschen" hervorzuheben. Was die Körperhaltung anbelangt, so werden die „alten Teutschen“ insgesamt statischer präsentiert, ohne Bewegung des Rumpfes, nur mit solcher der Hände, während die ,jetzigen Teutschen“ dynamischer gezeichnet sind: Sie sind mit dem gesamten Körper, einschließlich der Beine, in eine ausladendere Begrüßungsbewegung eingebunden. Mit diesen unterschiedlichen Darstellungen korrespondieren schließlich die unterschiedlichen Hintergrundszenarien, vor welche die Figurenpaare platziert sind: Die Silhouette einer Stadt mit Bürgerhäusern im gotisierenden Stil im ersten Fall, der Prospekt einer barockisierenden Palast- und Parkanlage im zweiten Fall, in deren Rundarkaden und Boskettenmuster sich die schwungvollen Kostümlinien der Vordergrundfiguren wiederfinden lassen.

Der Behauptungscharakter der Darstellung vertraut sichtlich auf die Aussagekraft des gewählten Frontispiz-Motivs im Kontext des Gesamtwerks - Kleidung, Körperauftritt und architektonische Formen werden hier als ein zeichenhaftes Ensemble präsentiert, in dessen Veränderung von „alt“ zu ,jetzig“" ein umfassenderer historischer Wandel wie in einem Prisma eingefangen ist und das von der geneigten zeitgenössischen Leserschaft entsprechend gedeutet werden kann.

Der Wandel, der hier vorgestellt wird, ist - vereinfachend und plakativ auf zwei Begriffe zugespitzt - der Wechsel vom älteren Stilideal der gravitas ${ }^{4}$ zu einem neuen Ideal der Leichtigkeit, ein Stilwandel, der an dieser Stelle nicht näher untersucht werden kann, der jedoch als mit den soziokulturellen, wissenschafts- und geistesgeschichtlichen Veränderungen vom 17. zum 18. Jahrhundert eng verschränkt verstanden werden muss. ${ }^{5}$

3 Ich nutze diesen Sammelausdruck, um auf das Ensemble zu verweisen, das sich aus der Haltung von Kopf, Rumpf, Armen und Beinen sowie aus der Orientierung von Körper und Gesicht auf ein Gegenüber ergibt. Dieses Ensemble erscheint in Abbildungen wie der hier und den im Folgenden besprochenen notwendig in Form einer Stillstellung zu Betrachtungszwecken - die darin eingefangene Bewegung und Dynamik des Körpers und seiner Gliedmaßen muss entsprechend extrapoliert werden, was notwendigerweise eine gewisse Unsicherheit der Interpretation mit sich bringt.

4 „Gravität“ ist im Umgangsdiskurs des frühen 17. Jh.s ein durchgängig positiv konnotierter Schlüsselbegriff, der in erster Linie auf körperliches bzw. körperkommunikatives Verhalten (v. a. auf Blick, Mimik, Gang) bezogen ist, jedoch auch verbales Verhalten (mit-)meinen kann.

5 Vgl. aber Linke (2010). 


\section{3. „Natürlichkeit“ vs. „Afectation: Neue Körperordnungen am Ende des 18. Jahrhunderts}

Das zweite Beispiel führt ins letzte Viertel des 18. Jahrhunderts und damit in eine Zeit zunehmender politischer, sozialer und kultureller Spannungen. Die Abb. 2 bis 4 kontrastieren ebenfalls wieder kommunizierende Paare. Die Doppelbilder - es handelt sich um Kupferstiche von Daniel Chodowiecki - gehören zu einer längeren Serie, sie entstammen dem Göttinger Taschenkalender auf die Jahre 1779 und 1780, wo sie unter dem Obertitel „Natürlichkeit vs. Afectation“"veröffentlicht wurden. Die Kupferstiche dieser Serie zeigen nicht immer, aber häufig Paare von Mann und Frau, wobei die Paare jeweils in derselben Situation, aber mit deutlich unterschiedlichem Körperauftritt dargestellt werden; und sie werden im Göttinger Taschenkalender begleitet von ebenso knappen wie kritisch-satirischen Kommentaren von Georg Christoph Lichtenberg.
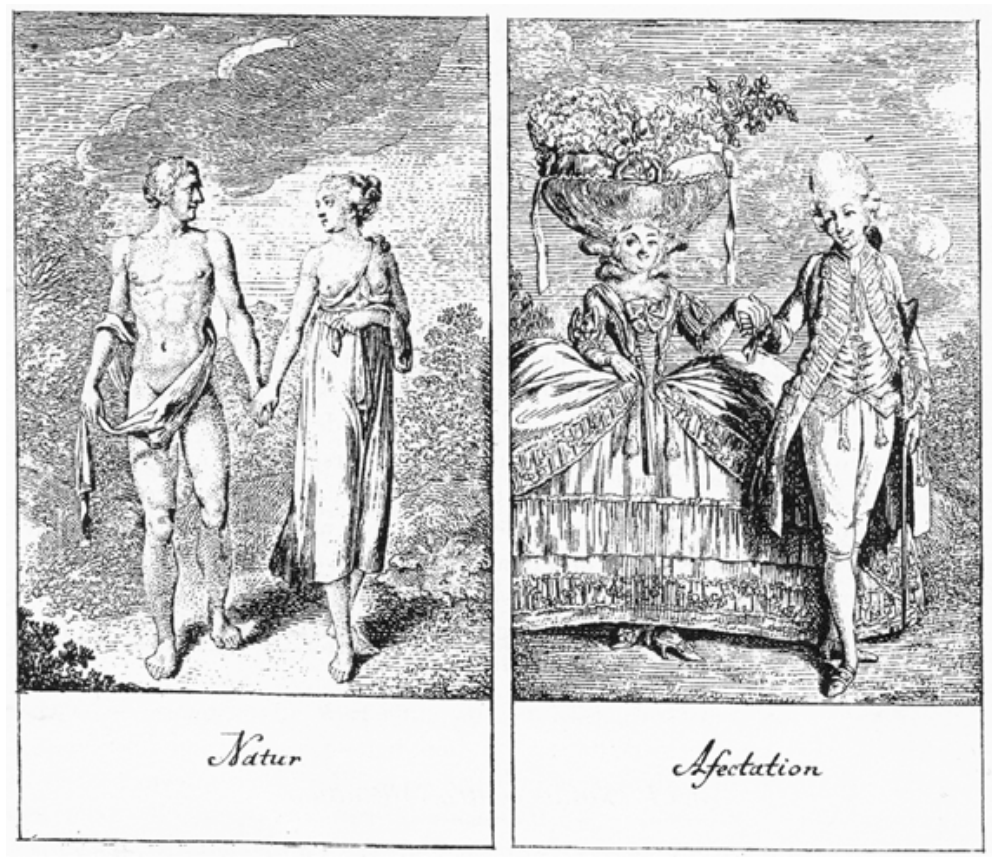

Abb. 2: Natur und Afectation, „Natürliche und affektierte Handlungen des Lebens“. Kupferstich von Daniel Chodowiecki. (Aus: Göttinger Taschenkalender auf das Jahr 1779 und auf das Jahr 1780) 

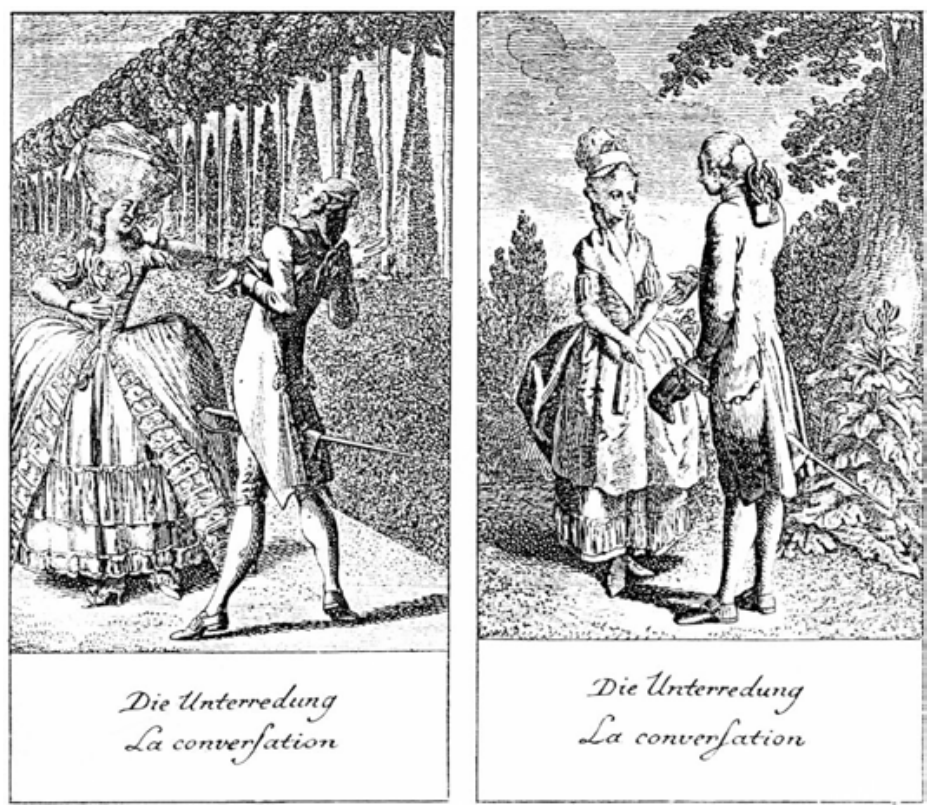

Abb. 3: Die Unterredung, La conversation. (Aus: Lichtenberg 1971, S. 37)
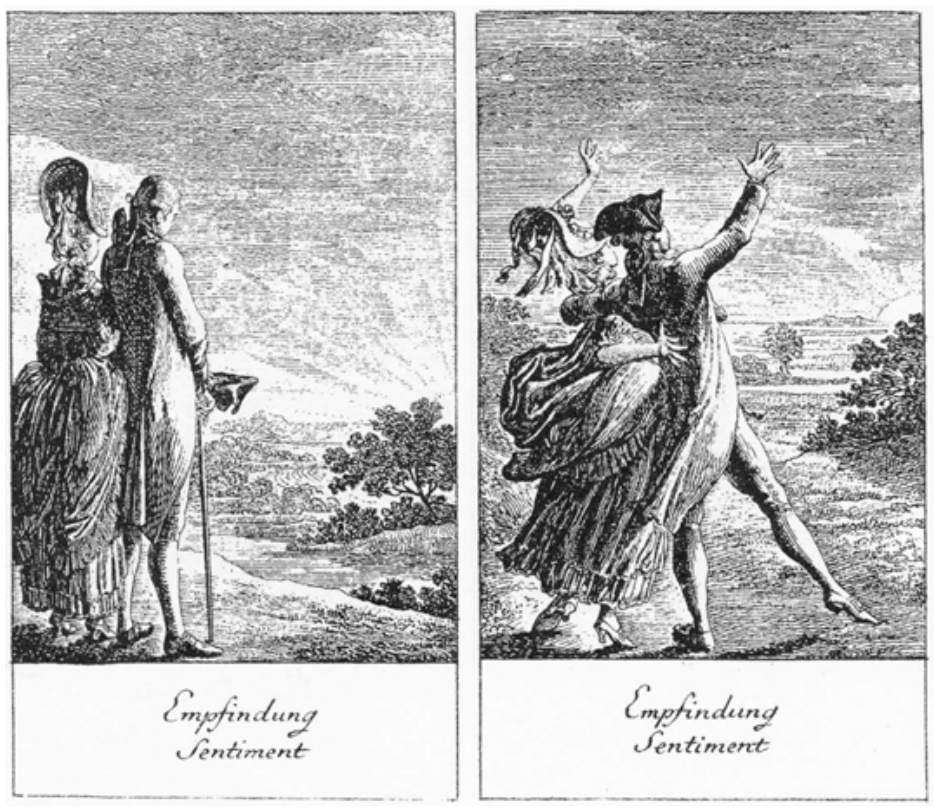

Abb. 4: Empfindung, Sentiment. (Aus: Lichtenberg 1971, S. 50) 
Abb. 2 zeigt das Paarbild, das den Titel der ganzen Serie - „Natürlichkeit vs. Afectation“ - illustriert, im zweiten Paarbild ist eine Gesprächsszene dargestellt und das dritte Bild schließlich bietet den Anblick zweier junger Paare, welche dem Naturschauspiel des Sonnenuntergangs hingegeben sind, allerdings unter sehr unterschiedlichem Einsatz ihrer Körperlichkeit.

Diese und die weiteren Bildpaare addieren sich zu einem Programm, das den epochalen Umschwung von einer adelsdominierten Gesellschaft zu einer bürgerlichen Gesellschaft in körpersemiotischer Verdichtung sowohl darstellt als auch kommentiert. ${ }^{6}$ Die einzelnen Bildpaare kontrastieren entsprechend ein im Körperhabitus adlig markiertes Paar mit einem bürgerlichen ${ }^{7}$, wobei

- Kleidung und äußere Erscheinung der „natürlichen“ Paare in jeder Hinsicht einfacher, die Kleider- und Körperlinien insgesamt schlanker und fließender sind,

- der bürgerliche gegenüber dem adligen Körperauftritt zurückgenommen erscheint und alles Ausladende wie auch alles allzusehr Bewegte vermeidet,

- und der als „natürlich“ apostrophierten bürgerlichen Körpersemiotik als korrespondierender Kontext eine freie Landschaft zugeordnet wird, während die adligen Figuren - wie noch die ,jetzigen Teutschen“ bei von Rohr - in barockisierende Gärten gestellt sind.

Diese Darstellung ,jetziger“ Deutscher vom Ende des 18. Jahrhunderts zeichnet sich also in erster Linie durch eine Zurücknahme des Körperauftritts, ja des Körpers selbst aus (und stellt damit gegenüber dem Körpermuster der ,jetzigen“ Deutschen bei von Rohr einen weiteren Paradigmenwechsel dar). In der Orientierung der Körper der bürgerlichen Paare aufeinander wird zudem ein privater und geschlossener Raum hergestellt, während die adligen Paare in ausgreifender und auf Zuschauer bzw. auf den Bildbetrachter ausgerichteter Gebärde den Raum öffnen und den Körper ausstellen. Dass in der Darstellung Chodowieckis (und im übrigen auch in den Kommentaren Lichtenbergs) Satire und Moral zusammenfließen, wird überdeutlich: einem als oberflächlich, theatralisch und affektiert charakterisierten Adel wird ein als ernst, selbstbeherrscht und „natürlich“ konzipiertes Bürgertum gegenübergestellt.

Wir haben es hier wohl noch mehr als in Abb. 1 mit einer normativen Bildaussage zu tun, mit einer Körperprogrammatik, die normativ ein Verhal-

6 Vgl. ausführlicher auch Linke (1996, S. 77ff.).

7 Ein Teil der Bilder ist aufgrund verschiedener Indikatoren auch so zu lesen, dass zwei bürgerliche Paare gegeneinander gestellt werden, wovon das eine sich allerdings an adligem Habitus orientiert bzw. diesen in grotesker Weise übersteigert. Vgl. hierzu etwa Scharloth (2005, S. 332-390). 
tensmuster erstellt, das in der vorgeführten Idealisierung und Kohärenz in der zeitgenössischen Realität sicher nicht zu finden war, die als Programmatik aber orientierenden Charakter und darin wiederum Langzeitwirkung hatte.

Auf was es mir jedoch in beiden Beispielen ankommt, ist, dass ein tiefgreifender sozialer und kultureller Umbau der Gesellschaft zeitgenössisch in programmatischer Weise an der Körpersemiotik festgemacht wird, dass die Stilistik der Leiblichkeit damit bewusst als Medium und Ausdruck soziokultureller Veränderungen wahrgenommen wird und dass sich also ,neue Zeiten' tatsächlich auch in einem Wandel der Sozialsemiotik des Körpers manifestieren.

Dies gilt auch für die gesellschaftlichen und kulturellen Veränderungen der 1968er Jahre.

\section{1968: Entdifferenzierungen}

$\mathrm{Zu}$ den körperbezogenen Elementen, mit welchen sich die protestierenden Jugendlichen und Studierenden, für die später das Label Achtundsechziger üblich werden wird, von der älteren und damit auch Eltern-Generation abgrenzten und die sie in deren Augen wiederum als ebenso irritierend wie auch als politisch-ideologisch und alltagsmoralisch irregeleitet erscheinen ließen, gehörten u.a. die neue Uni-Form von Jeans und Pullover, die als ,Gammler-Look ${ }^{6}$ terminologisierte neue Nachlässigkeit in der Zurichtung von Kleidung, Haupt- und Barthaar, der Verzicht auf eine ,ordentliche Einrichtung von Zimmern und Wohnungen sowie als unanständig oder gar ,vulgär ${ }^{夭}$ empfundene Formen des körperlichen Auftritts in öffentlichen oder gar offiziellen Situationen. Als diesen unterschiedlichen Anstössigkeiten gemeinsam erweist sich der Aspekt der Entdifferenzierung, und zwar sowohl mit Blick auf die vertikalen wie horizontalen Achsen alltäglicher Lebenswelt und ebenso in ganz konkreter wie in metaphorischer Bedeutung. ${ }^{8}$

Auf Kleidung und Äußerlichkeiten des Körpers werde ich im Folgenden nicht weiter eingehen, obwohl sich gerade darin das, was man aus gegenwärtiger, rückblickender Perspektive als den semiotischen Code der 68er empfindet, vielleicht am plakativsten festmachen lässt ${ }^{9}$ und die auch in diesem semiotischen Feld beobachtbaren Entdifferenzierungen zu dem die Zeitgenossen irritierenden Gesamteindruck der protestierenden Jugend in

8 Vgl. zu diesem Begriff Link (2001), ich komme unter Kap. 5 ausführlicher auf die Verwendung des Begriffs bei Link zurück.

9 Vgl. hierzu die für die angesprochene Thematik wichtige Studie von Grob (1985), deren zweiter Teil (Kap. 9) sich mit dem „Kleidungsverhalten innerhalb der Studentenbewegung“ befasst. 
hohem Maße beigetragen haben dürften. Beispiele für solche Formen der Entdifferenzierung in der Mode wie in den Bekleidungspraktiken sind etwa die Annäherung von männlicher und weiblicher Kleidung (hier plakativ die Aufnahme der Hose in die weibliche Alltagskleidung ${ }^{10}$, aber auch die Aufnahme „weiblich“ konnotierter Farben, Muster und Schnitte in die Männermode $\left.^{11}\right)$ sowie die Annäherung von männlicher und weiblicher Haartracht (d.h. neue Länge und neue Schnitte in den Männerfrisuren, prominent in den aus heutiger Perspektive eher als unauffällig empfundenen „Pilzköpfen“ der Beatles) sowie auch der demonstrative Verzicht auf situationskontextualisierende Kleidung, wie dies etwa für die traditionell deutliche Unterscheidung von Werktags- und Sonntagskleidung bzw. von Freizeit- und Berufskleidung galt.

Ich möchte dieses weite Feld hier jedoch nicht weiter ausleuchten, dagegen zwei etwas weniger klar fassbare, jedoch gerade deshalb vielleicht besonders irritierende Formen des Körperauftritts näher thematisieren, nämlich neue Formen des Sitzens und des Liegens, sowie auf eine Entdifferenzierung in der funktionalen Topographie des privaten Raumes eingehen. Alle drei genannten Phänomene, das ,neue Sitzen', das ,aktive Liegen' sowie die neue Alltagstopographie, erachte ich als konstitutive Elemente eines neuen Lebensgefühls, eines neuen Selbstverhältnisses wie eines neuen Verhältnisses von Privatheit und Öffentlichkeit, das die 68er auszeichnet.

\subsection{Neues Sitzen}

Das Sitzen, vor allem das Auf-dem-Boden-Sitzen, kann als ein zentrales Element im Körpercode der revoltierenden Studierenden wie auch ihrer weniger bewegten Generationsgenossen gelten, und zwar sowohl in mehr privaten wie in mehr öffentlichen Spezifizierungen.

Das unter dem Label „Sit-in“ fungierende, öffentliche Am-Boden-Sitzen als Körpergeste der Raumbesetzung wie auch als konkreter Stör-Einsatz des Körpers zur Blockade von Durchgängen und Wegen ist in den 68er Jahren zunächst auf studentische Protestaktionen bzw. auf den universitären Kontext beschränkt (vgl. Abb. 5), entwickelt sich dann jedoch in den folgenden Dekaden zu einer allgemein genutzten Form des (in erster Linie linken) Protestes, nicht zuletzt etwa im Kontext von Anti-Atomkraft sowie AntiAufrüstungs-Demonstrationen (Abb. 6).

10 Vgl. auch Grob (1985, S. 225f.).

11 Vgl. auch Grob (1985, S. 229f.). 


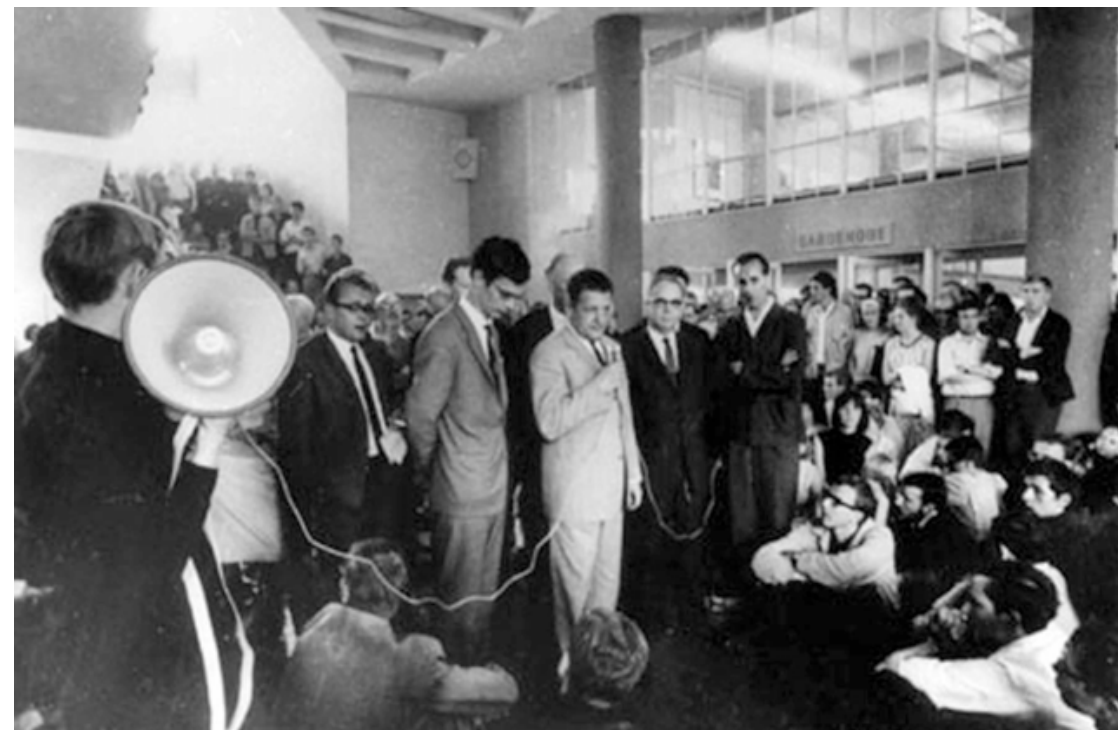

Abb. 5: 22.6.1966. Sit-in von 3000 Studierenden im Henry-Ford-Bau, HSA FUB, Bildarchiv der Zeitschrift Colloquium. (http//web.fu-berlin.de/chronik/b-picts/1961-1969/sitin.html, 14.4.2008)

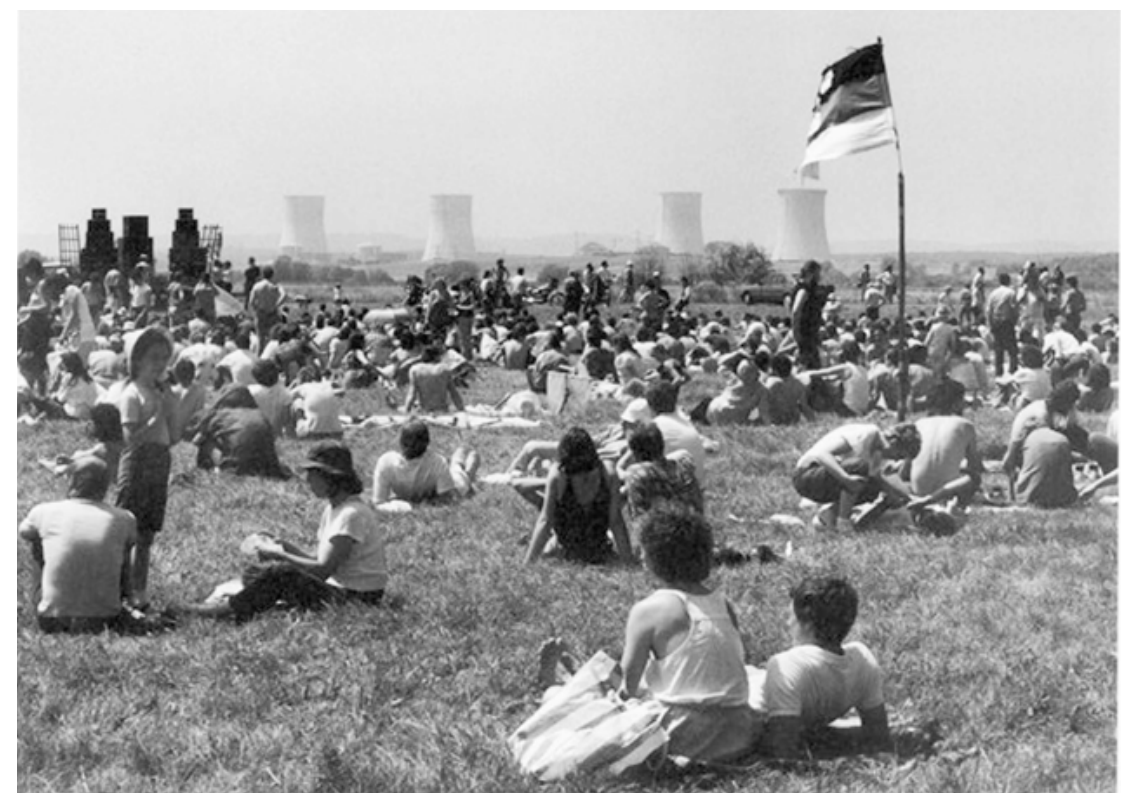

Abb. 6: Demonstration gegen taktische Atomwaffen, Fulda 1984. (Aus: Klemm 1999) 
Daneben gibt es das Sich-auf-den-Boden-Setzen in öffentlichen und balb-öffentlichen Kontexten, in denen traditionell das Sitzen auf Stühlen oder eine Form von Stehen als angemessen empfunden worden wäre. Dies gilt für universitäre Veranstaltungen in überfüllten Hörsälen (Abb. 7) ebenso wie für Popkonzerte. Mit Blick auf letztere ist es also nicht nur die Musik selbst, die sie von klassischen Konzerten unterscheidet, sondern auch andere Praktiken des Sitzens (die ihrerseits wieder sowohl mit neuen Bekleidungsformen verbunden sind, d.h. mit Kleidern, deren Material ein Am-BodenSitzen zulässt, wie dies prototypisch bei Jeans der Fall ist, als auch mit einer neuen Nachlässigkeit gegenüber, Gebrauchsspuren' an Kleidern ${ }^{12}$ ). Dass im Anschluss an ein Konzert der Rolling Stones 1967 in Zürich ausgerechnet das vorhandene Stuhlmobiliar zum Gegenstand eines ungesteuerten Aggressionsausbruchs gemacht und zum größten Teil zerstört wurde - eine Objektwahl, die Polizei wie Presse eher ratlos zur Kenntnis nahmen (vgl. Abb. 8) - mag in diesem Kontext zeichenhaft sein.

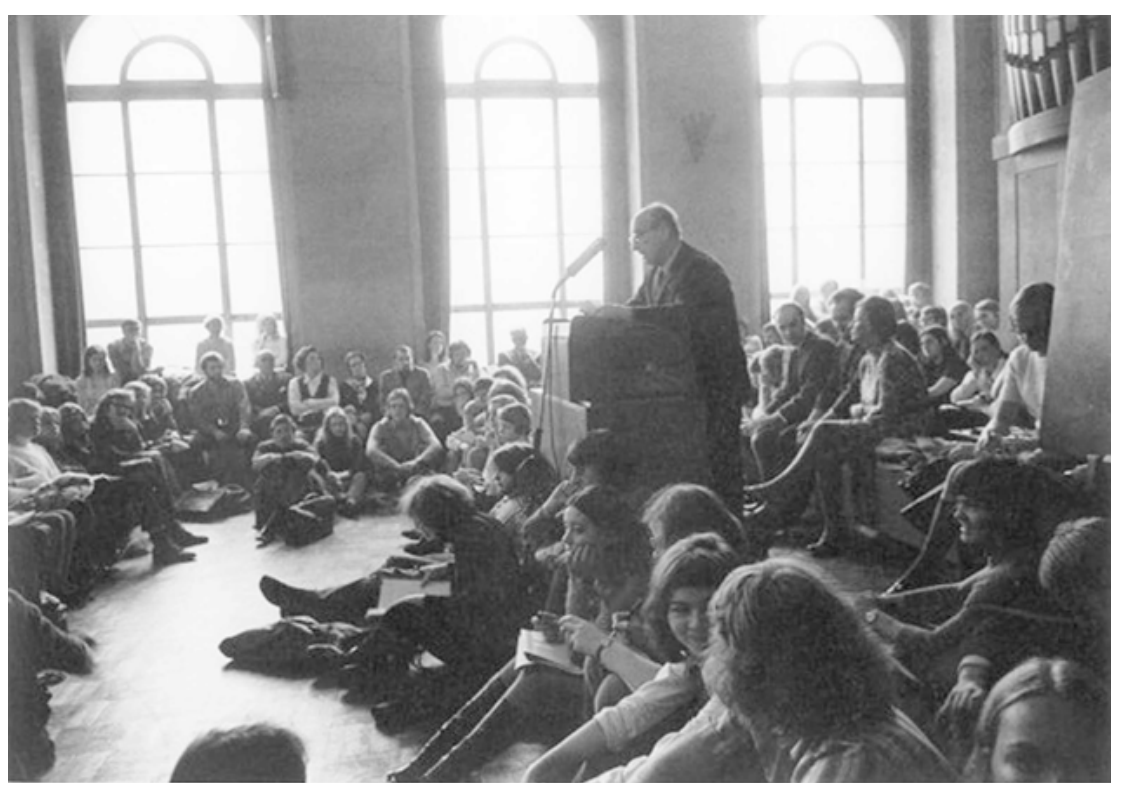

Abb. 7: Alexander Mitscherlich, Universität, Frankfurt 1970. (Aus: Klemm 1999)

12 Vgl. Grob (1985, S. 260ff.). 


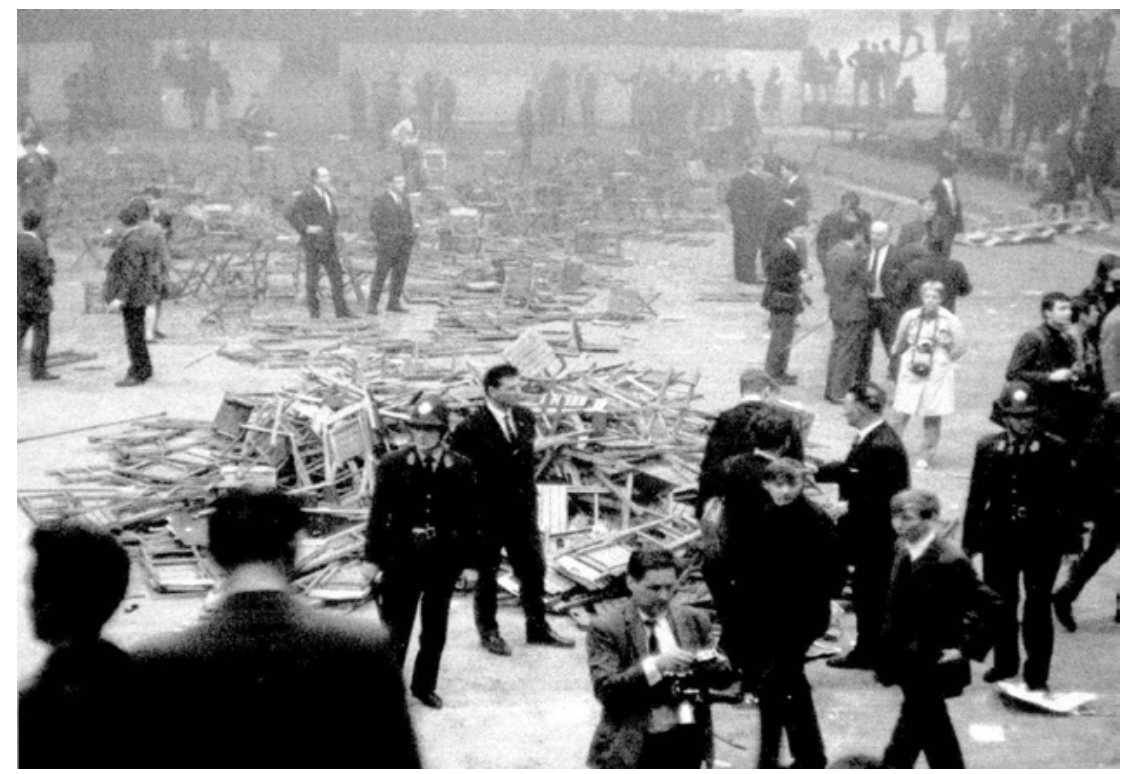

Abb. 8: Das Zürcher Hallenstadion nach dem Konzert der Rolling Stones, 1967. (Aus: Linke/Scharloth 2008, S. 103)

Im privaten Bereich bildet das (gesellige wie einsame) Auf-dem-Boden-Sitzen in den 60er Jahren einen Generationencode, der zweifellos in einem Resonanzverhältnis zum öffentlichen Auf-dem-Boden-Sitzen steht, diesem aber unter Umständen sogar zeitlich vorausgeht und nicht auf die politisierten Gruppierungen beschränkt ist. Dieses ,neue Sitzen` ist besonders dann als aktive, semiotisch signifikante Handlung markiert, wenn es in klassisch möblierten Wohnräumen stattfindet, in denen Stühle oder andere Sitzflächen durchaus zur Verfügung stehen. Ein zeitgenössisches Anstandsbuch vermerkt diese ,neue' Form von Raumaneignung als Körpergeste zur Markierung von Ungezwungenheit, die aber durchaus akzeptabel sei, sofern sie „mit Anstand“ erfolge und auf bestimmte situative Settings (hier wird explizit die „Party jüngerer Leute“ genannt) beschränkt bleibe (vgl. die Darstellung sowie den entsprechenden Begleittext in Abb. 9). Durch diese Zuschreibung einer situationskontextualisierenden Funktion wird einerseits der mögliche gesellschaftskritische Aspekt des neuen Sitzens, der sich gegen bestehende Körperordnungen wendet, entschärft, andererseits und gleichzeitig wird damit aber auch - sozusagen als nicht intendierter Neben-Effekt dieser Argumentationslinie - der Interaktionsmodus der Informalität aufgewertet. 


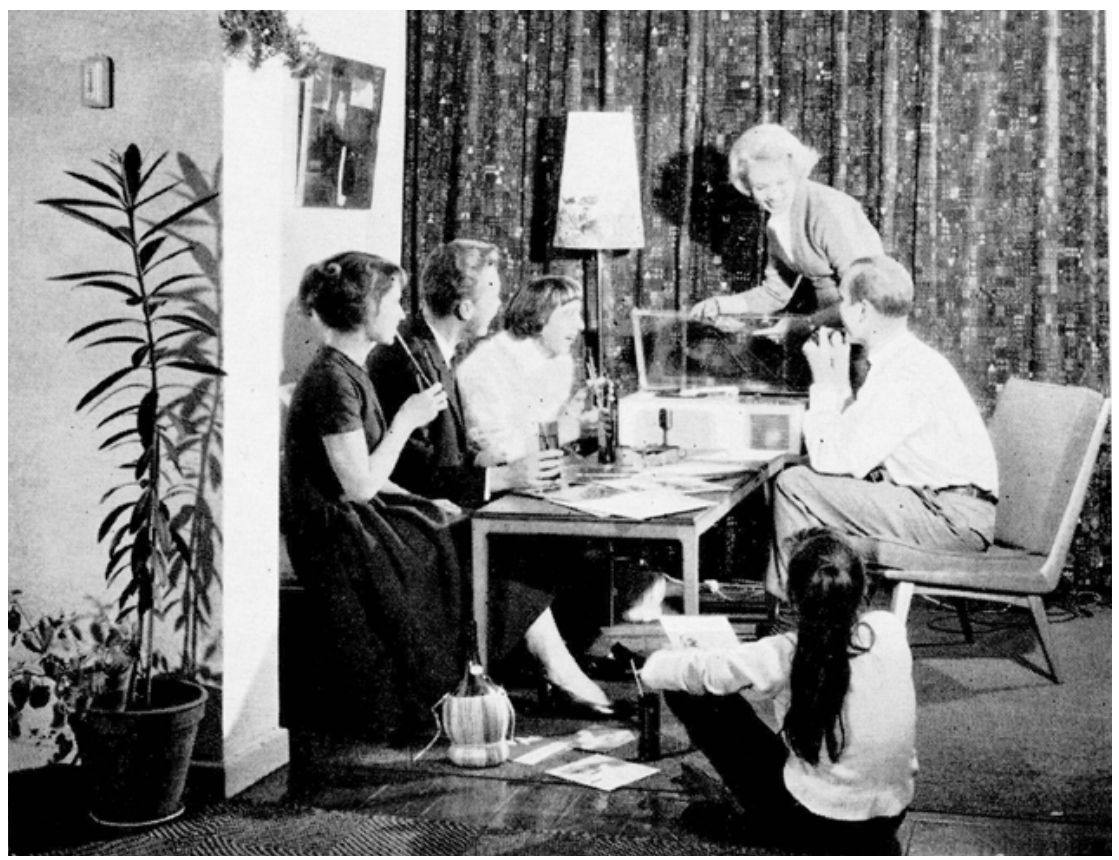

Abb. 9: „Bei einer Party jüngerer Leute geht es heute ganz ungezwungen zu. Da darf man ruhig auch mal auf dem Fußboden sitzen, wenn man es mit Anstand tut.“ (Aus: Haller 1970, S. 145)

Eine Stabilisierung bzw. Hegemonialisierung dieses situationskontextualisierenden Auf-dem-Boden-Sitzens findet sich schließlich dort, wo schon die Einrichtung von Privatträumen darauf hin angelegt ist, d.h. wo auch die vorgesehenen Sitzmöbel in erster Linie dazu dienen, das Sitzen auf dem Boden etwas weicher zu gestalten, und nicht mehr dazu, den Körper über den Boden zu erheben - vgl. hierzu die Abb. 10 des, Wohnzimmers' der Berliner Kommune 1 (Rainer Langhans beim Telefonieren). Dabei ist festzuhalten, dass solche ,Möbel' für das Sitzen auf dem Boden vielfach Bricolage-Charakter haben, d.h. es handelt sich oft um Matratzen oder Kissen, die über ihren ursprünglichen Gebrauchskontext mehr eine liegende als eine sitzende Körperhaltung konnotieren. 


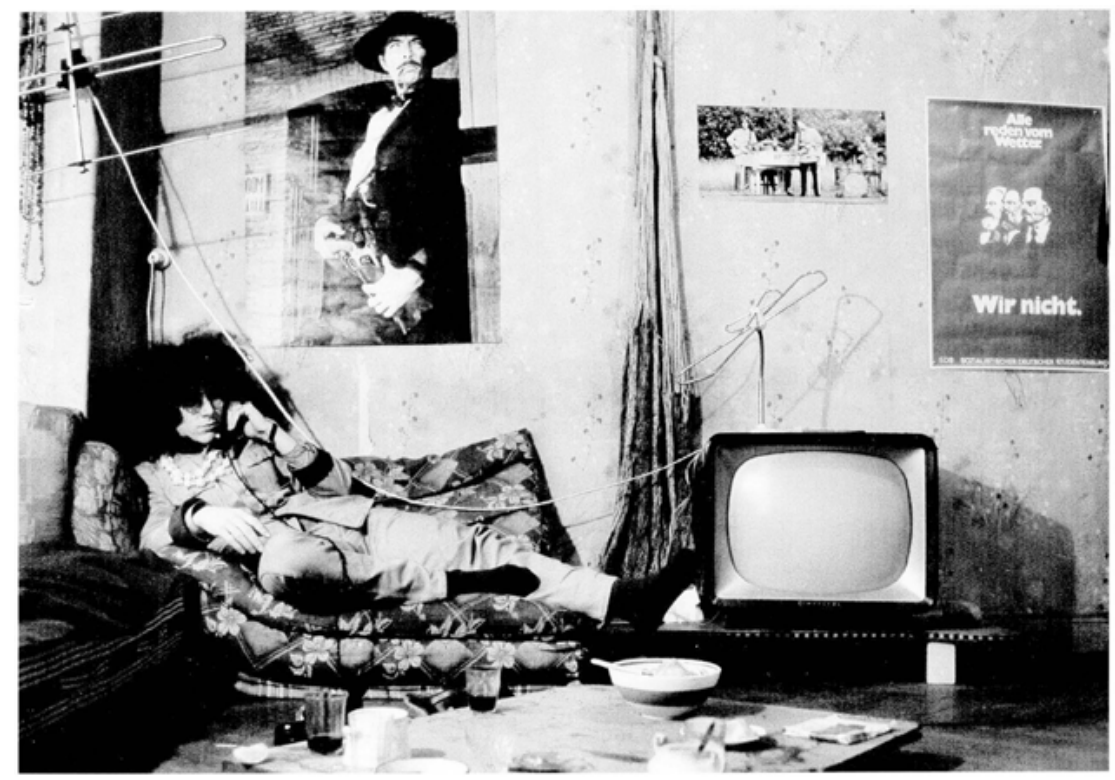

Abb. 10: Pascha. 1968. (Aus: Ruetz 1997, S. 280)

An diesem Punkt wäre eine ausführlichere Beschäftigung mit dem zeitgenössischen Möbeldesign lohnend, die hier aber nicht geleistet werden kann. Ganz allgemein lässt sich aber zumindest festhalten, dass auch im Design von Sitzmöbeln die Aufweichung der klassischen Sitzhaltung angelegt ist, insofern als neue Sofa- und Sesselformen auch neue Körperhaltungen evozieren und diese damit zu ,angemessenen' Haltungen machen: Die Parallelen zwischen Protestsemiotik und Designavantgarde sind auffällig (Abb. 11 und 12). Allerdings: Das ,neue' Sitzen auf Matratzen und Kissen, d.h. auf improvisierten Unterlagen, die gerade nicht von sich aus auf Stützung und Formung des Körpers bzw. bestimmter Sitzhaltungen angelegt sind, markiert in erster Linie einen deutlichen Bruch gegenüber tradierten Sitzpraktiken, d.h. wir haben es beim ,neuen Sitzen'zunächst mit einer Kontrastgeste zu tun, die die Selbständigkeit und Freiheit des Körpers betont, während mit dem neuen Möbeldesign, das zudem oft in futuristische Entwürfe höhlenartiger Wohnungsausgestaltung eingebettet ist, der Versuch einer neuartigen harmonischen Synthese von Körper und Möbel angestrebt wird. Diese Gleichartigkeit des Ungleichen erzeugt eine Spannung, der nachzugehen lohnend wäre und lässt in jedem Fall den Körpercode der 68er als Teil einer allgemeineren Entwicklung erscheinen, welche die hergebrachten Körperordnungen in Frage stellt. 


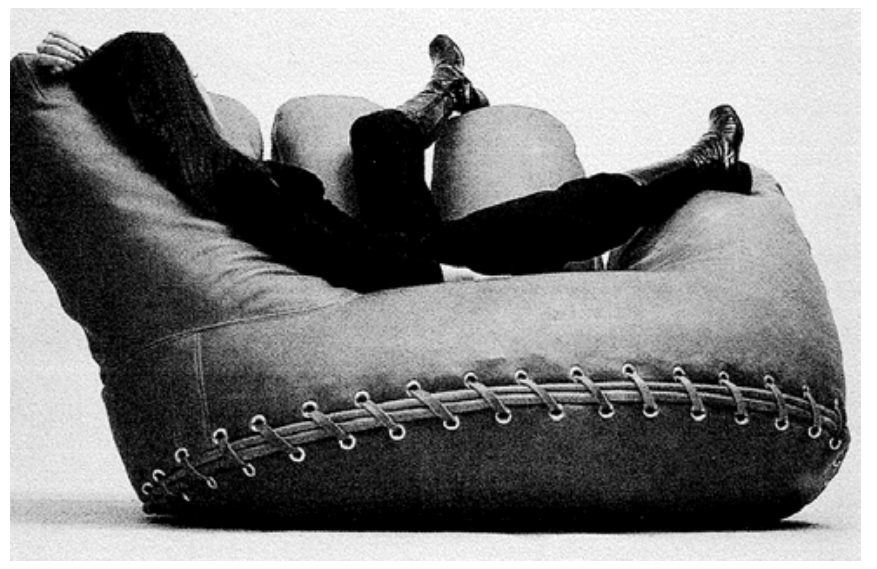

Abb. 11: Sofa. Joe, 1970. Jonathan de Pas/Donato D’Urbino/Paolo Lomazzi. Poltronova. (Aus: Breuer/Peters/Plüm 1999, S. 128)
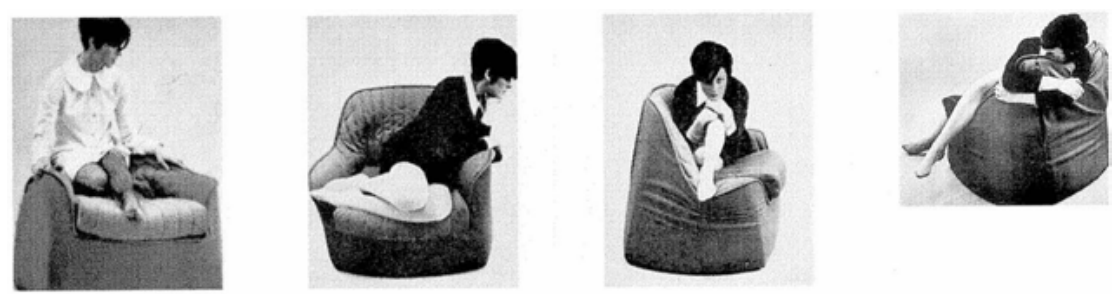

Abb. 12: Sessel, 1968, Afra und Tobias Scarpa. (Aus: Breuer/Peters/Plüm 1999, S. 127)

\subsection{Aktives Liegen}

Das Liegen als „öffentliche“ Körperhaltung hatte bis ins 18. Jahrhundert hinein zumindest im Rahmen fürstlichen Hofzeremoniells einen akzeptierten $\mathrm{Ort}^{13}$; in der historischen Folge und außerhalb zeremonieller Kontexte findet es sich - in Innenräumen - lediglich in einigen wohldefinierten soziokulturellen Nischen wie etwa in der Salonkultur des späteren 18. und beginnenden 19. Jahrhunderts als weibliche Position des Liegens beim Empfang informellerer Besuche (dann ebenfalls auf entsprechenden Spezialmöbeln wie der Récamiere ${ }^{14}$ ) oder - in Außenräumen - als sozialrepräsentativ markierte (und ideologisch aufgeladene) Pose des In-der-Natur-Ruhens. Auch heute, d.h. unter gegenwärtig gültigen Körperordnungen, ist das Liegen außer-

13 Hierher gehört zentral die Situation des (fürstlichen) Lever, d.h. des Empfangs von Besuchern in den Schlafräumen vor und während des Aufstehens.

14 Diese Liegeposition zeigt auch das berühmte Gemälde Jacques-Louis Davids von Julie Récamier auf dem nach ihr genannten Möbel. 
halb entsprechend definierter Räume (wie etwa Freibad und Strand) und Domänen (Freizeit) keine öffentlichkeitstaugliche Körperhaltung; selbst im privaten Bereich der eigenen Wohnumgebung gilt - außerhalb von Krankheitsfällen - eine liegende Haltung in der Gegenwart von anderen als äuBerst informell und an der Grenze zur Unhöflichkeit.

Nun lassen sich aber gerade in den 68er Jahren ,aktive ${ }^{6}$ und in ihrer Abweichung von gängigen Körperordnungen auch entsprechend demonstrative Formen des kommunikativen Liegens beobachten, wie etwa in Abb. 13 aus dem Alltag der Kommune 1 in Berlin, in der Fritz Teufel sich sogar sichtlich aktiv anstrengt, um in eine liegende Haltung zu geraten. Auch gegenwärtigem Normempfinden dürfte diese Haltung als markiert auffallen.

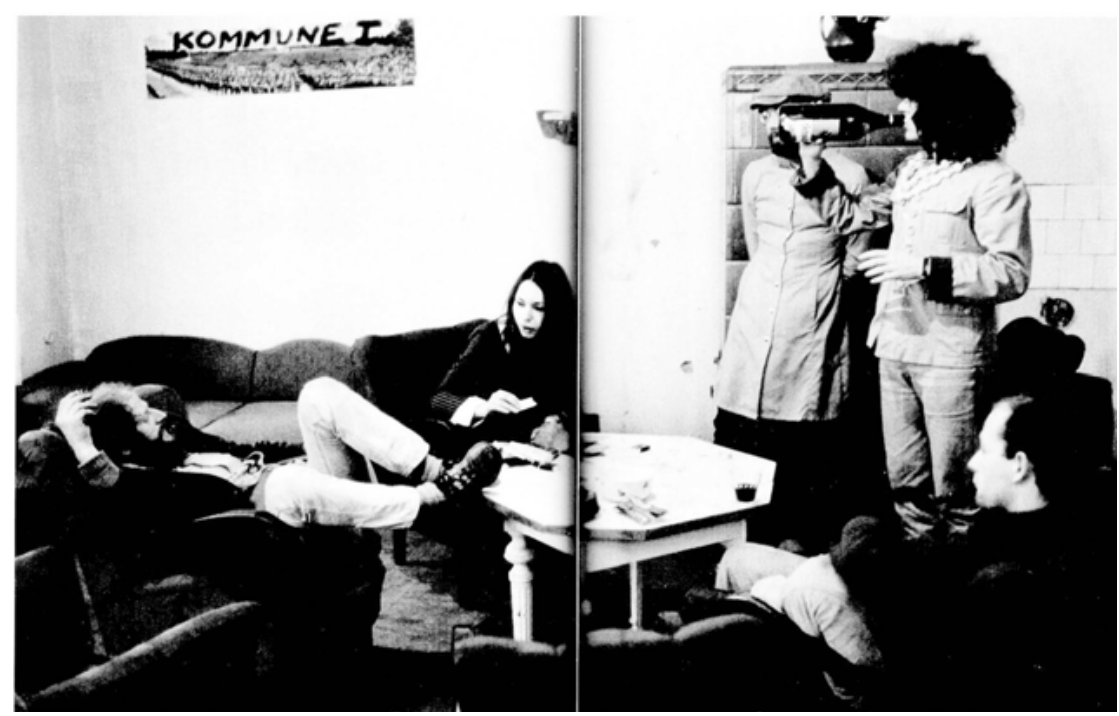

Abb. 13: Die Bohème. Szene aus der Kommune 1. Berlin-Charlottenburg, Stuttgarter Platz. 8. März 1968. (Aus: Ruetz 1997, S. 154-155)

Dies gilt wohl in noch stärkerem Maße für Abb. 14, die einen auf einem Tisch liegenden Studenten zeigt. Tisch samt Student befinden sich im Rektorat der Freien Universität in Berlin, die Szene spielt sich im Rahmen einer Besetzung der Rektoratsräume der FU ab, und dass die anwesenden Polizisten auf normalen Stühlen sitzen, markiert sowohl die Liegehaltung des Studenten sowie den Liegeort noch zusätzlich als abweichend. Auch zu dieser Form des Auf-dem-Tisch-Liegens finden sich - wie schon beim Aufdem-Boden-Sitzen - Parallelen im Bereich privater Geselligkeit, wie Abb. 15 aus einem Anstandsbuch von 1970 zeigt. Auch hier wird dem ,neuen Liegen' eine kontextspezifische Funktion bzw. eine situationskontextuali- 
sierende Potenz zugeschrieben, indem darauf verwiesen wird, dass die Szenerie eben das „bei jungen Leuten so beliebte Kellerfest“ (Haller 1970, S. 177) auszeichnet. ${ }^{15}$ Zusätzliche semiotische Signifikanz wird auch erreicht, wenn ,aktives Liegen' auf der Straße und damit in Staub und Dreck stattfindet wie in Abbildung 16 (Weltjugendfestspiele DDR 1973). Hier wird die Unabhängigkeit der gewählten Haltung von ansonsten als nötig gedachten Voraussetzungen wie etwa einer weichen oder zumindest sauberen Unter-

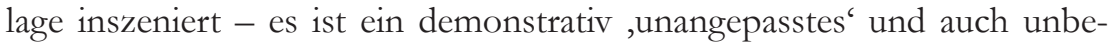
quemes Liegen, das gerade deshalb als semiotisch signifikant interpretiert werden muss.

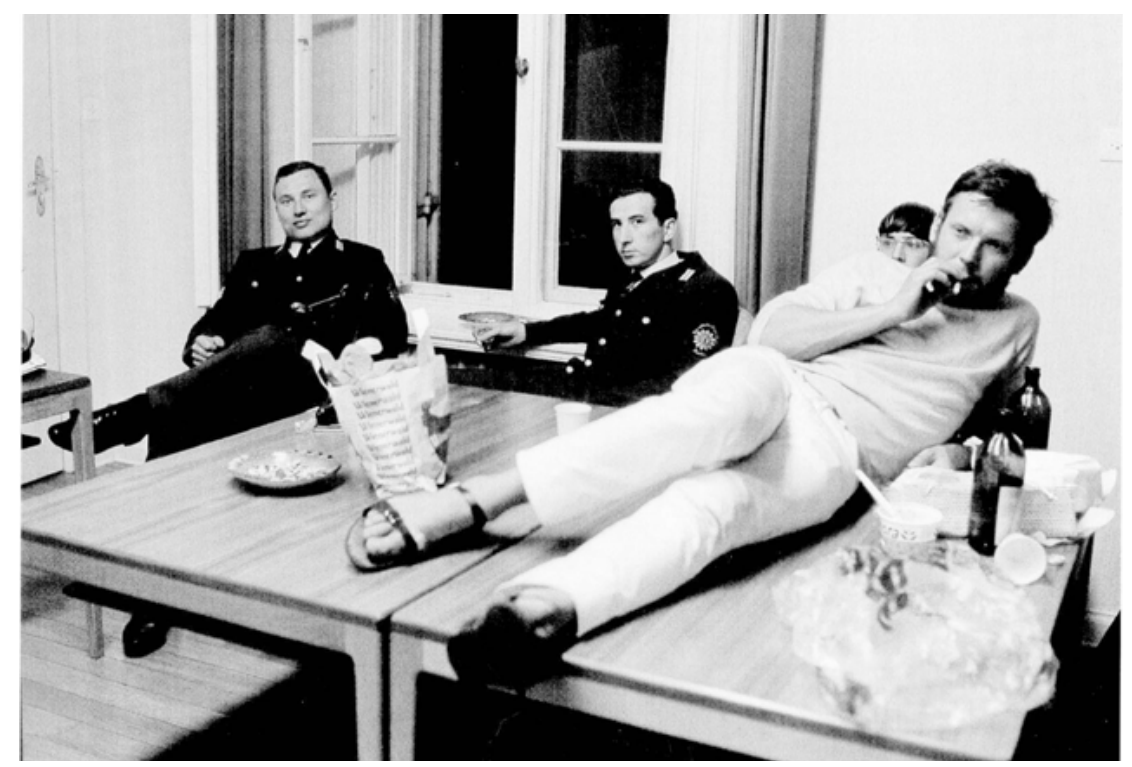

Abb. 14: Revolutionäre Ruhe. Besetzung des FU-Rektorats. Berlin-Dahlem. 27. Juni 1968. (Aus: Ruetz 1997, S. 233)

15 Die ,Nähe zum Boden“ und damit eine „Entvertikalisierung“ der Raumnutzung zeigt sich grade auch auch in dieser Aufwertung von Kellerräumen zu Gesellschaftsräumen im sprichwörtlichen „Partykeller“ der 60er und 70er Jahre, der im Kontrast steht zum Ballsaal der Beletage und der den Souterrain, der als Kellerwohnung bis weit ins 20. Jahrhundert hinein sozial markiert ist, bis zu einem gewissen Punkt kulturell neu definiert. 


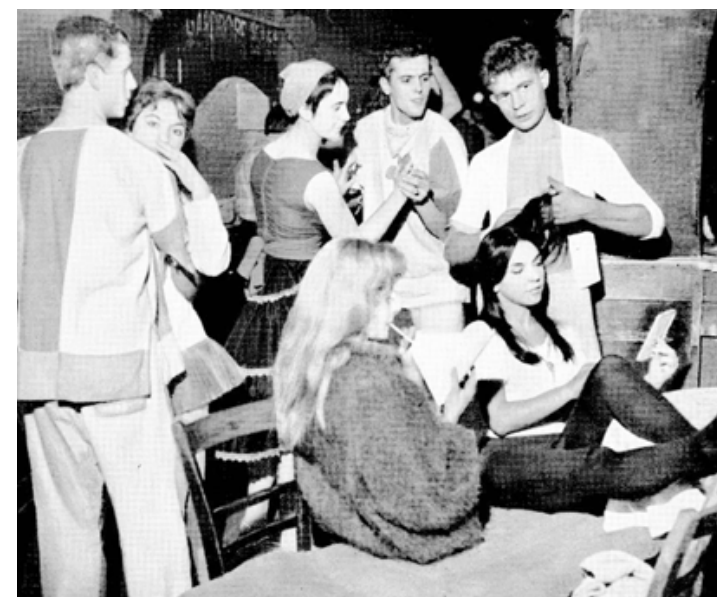

Abb. 15: Kellerfest. (Aus: Haller 1970, S. 177)

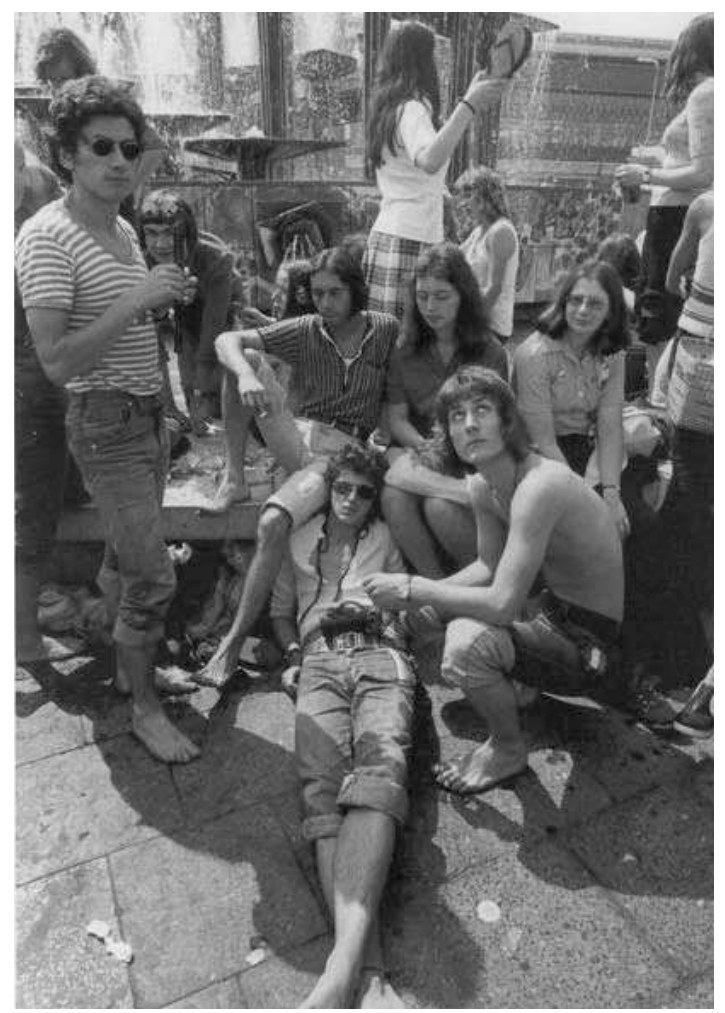

Abb. 16: Weltjugendfestspiele, Ost-Berlin 1973. (Aus: Klemm 1999) 
Auch Abb. 17 zeigt eine Variante solchen ,aktiven Liegens'. Diese Abbildung dokumentiert im übrigen, dass die liegende Position als Element der körpersemiotischen Selbstformierung einer ,neuen' Generation der spezifisch protestsemiotischen Nutzung dieser Haltung nicht erst nachfolgt, sondern zumindest parallel $\mathrm{zu}$ ihr funktionalisiert wird: Die Abbildung stammt aus einem 1967 publizierten Anstandsbuch ${ }^{16}$ und belegt damit, dass diese Haltung bereits zu diesem Zeitpunkt als Element eines jugendlichen Körpercodes wahrgenommen wurde.

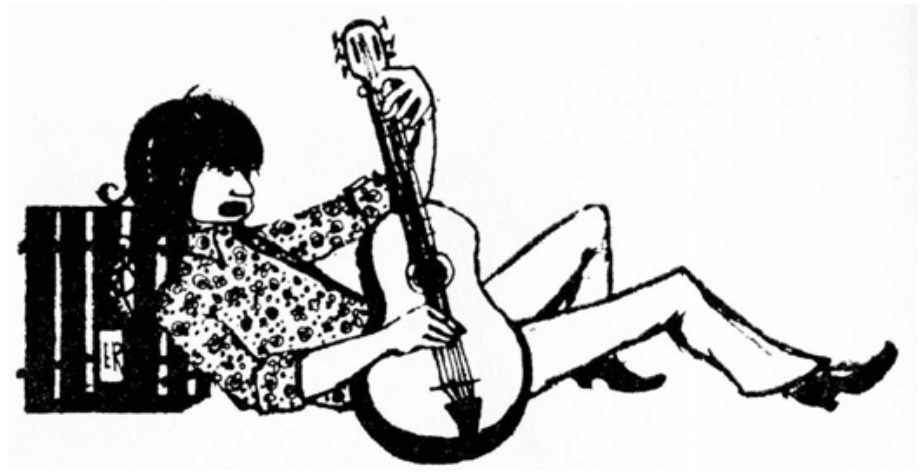

Abb. 17: Liegender Gitarrenspieler. (Aus: Schittenhelm 1967, S. 130)

Wie im übrigen auch die Haltung des Gitarrenspielers zeigt, handelt es sich in all den vorgeführten Liegehaltungen um ein Liegen mit aufgestütztem Oberkörper, was dieses Liegen deutlich als ein kommunikatives und damit eben auch ,aktives' charakterisiert: Beim völlig flachen Liegen ist die Frontalorientierung gegenüber einem Kommunikationspartner, die als Schlüsselsignal für die Aufnahme kommunikativen Kontaktes dient, nicht mehr möglich. Der Liegende ist dann darauf angewiesen, dass sein Gegenüber diese Orientierung herstellt (z.B. indem er sich über ihn beugt), er würde sich dadurch also selbst auf eine markiert passive Rolle im kommunikativen Austausch beschränken. Dies gilt für das ,aktive Liegen' gerade nicht.

Insgesamt zeigt sich in diesen Sitz- und Liegehaltungen eine aktive Entdifferenzierung in der vertikalen Achse von Raum- und Körperordnungen. Die Distanznahme vom Boden beim Sitzen und Liegen als durchgängiges Merkmal westlicher Raumordnung sowie die vertikale Ausrichtung des Körpers, die mit einer sozialsemiotischen Aufladung der aufrechten Haltung eng verknüpft ist und die beide Normalformen (sensu Jürgen Link) von Raum und Körperlichkeit darstellen, werden durch die ,neuen' Körperhaltungen sowie die betonte Orientierung nach unten als soziokulturell konstituierte Ordnungen bewusst gemacht und der mit ihnen verbunde-

16 Schittenhelm (1967, S. 130). 
ne (bzw. ihnen zumindest unterstellte) Disziplinierungscharakter auf diese Weise gleichzeitig markiert und durchbrochen.

\subsection{Entdifferenzierung von Topographien}

Die konkrete wie semiotische Aufwertung der Nähe zum Boden, die als Teil einer Raum-Körper-Programmatik der Jugendgeneration der 60er und frühen 70er Jahre gelten kann, zeigt sich auch in Abb. 18. Das Bett in Form einer Matratze, die direkt auf dem Boden liegt, dürfte für einen großen Prozentsatz zumindest der studentischen Jugend in den späteren 60er Jahren und noch weit in die 70er Jahre hinein Normalität gewesen sein - es ist eine generational und sozial markierte Normalität.

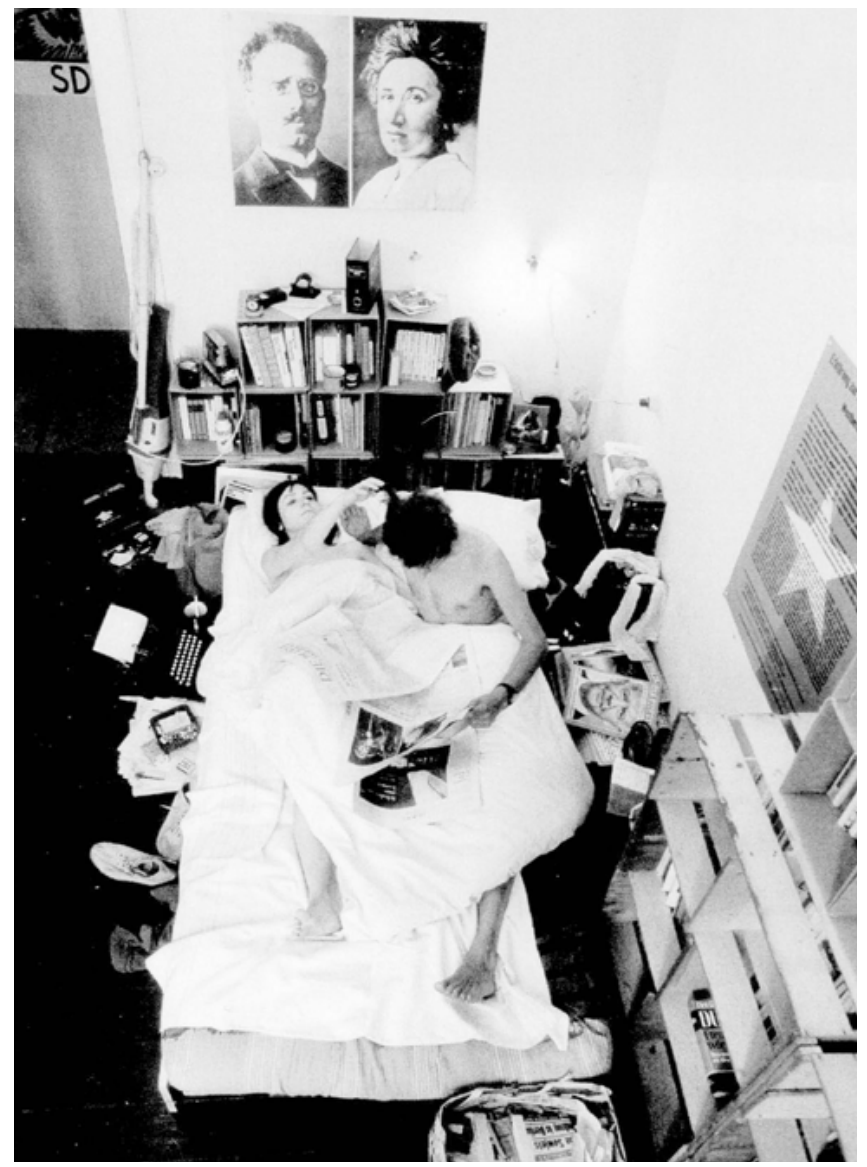

Abb. 18: Nachtlager. Studentenbude. Berlin-Charlottenburg. 12. September 1968. (Aus: Ruetz 1997, S. 150) 
Drei (eng miteinander verknüpfte) Aspekte scheinen mir für die hier wiedergegebene Raumszene bzw. Rauminszenierung ${ }^{17}$ semiotisch besonders signifikant:

- Das Bild dokumentiert einen neuen Blick auf Sexualität: Ein Paar, offensichtlich nackt, im Bett, wird hier in seiner unspektakulären Alltäglichkeit vorgeführt und im Bild dem Blick von Dritten zugänglich gemacht, ohne dass damit pornographische Konnotationen erzeugt werden (sollten). Das Bild steht in seiner Zeit zudem nicht allein: Es evoziert vielmehr weitere ähnliche Bilder aus den 68er Jahren, darunter prominent auch diejenigen von John Lennon und Yoko Ono bei ihrem „Bed-In“ 1969 zunächst im Hilton-Hotel in Amsterdam und dann nochmals in Montreal. ${ }^{18}$

- Das Bett selbst ist hier nicht in erster Linie als erotisch oder sexuell konnotierter Ort konstruiert, sondern erscheint als ein multifunktionaler Aufenthaltsort, an den sich verschiedene Lebensdomänen anlagern: Die im Bett und ums Bett herum versammelten Gegenstände - von der Zeitung über Bücher, Essensreste, Aschenbecher, Kaffeetassen, Schreibmaschine, Kleider, Schuhe bis zum Staubsauger kontextualisieren so unterschiedliche Aktivitäten wie Schlafen, Lesen, Essen, Schreiben, Rauchen, Putzen und Kaffeetrinken, also Kontexte und Aktivitäten, denen ansonsten - vor allem im Rahmen von traditionellen Mittelschichtsszenarien - unterschiedliche Räume oder zumindest durch Möblierung und andere semiotische Anstrengungen klar getrennte Bereiche innerhalb ein und desselben Raumes zugeordnet sind.

- Die Abbildung dokumentiert damit eine horizontale Ent-Differenzierung alltäglicher Lebenswelt, d.h. sie zeigt die Auflösung einer hergebrachten Wohnraum-Topographie, deren Ordnungen und Grenzziehungen eng mit Rollenverständnissen, Tagesstrukturen und der soziokulturellen Bewertung von alltäglichen Aktivitäts- und Handlungstypen zu tun haben. Zeitgenössischen Betrachtern (nicht nur, aber ganz bestimmt solchen der älteren Generation) dürfte die abgebildete Raumszene als dezidiert unordentlich vorgekommen sein. ${ }^{19}$ So ist etwa die Schreibma-

17 Die mögliche Gestelltheit des Bildes (M. Ruetz kommentiert dies in seinem Bildband nicht) tut seinem Belegwert keinen Abbruch, insofern Gestelltheit hier weitgehend mit Programmatik zusammenfallen würde (und es in erster Linie diese ist, die hier interessiert). Zudem dürfte das Bild bei Zeitgenossen - abgesehen von den individuellen Personen, die abgebildet sind - einen hohen Wiedererkennungswert besitzen: In Wohngemeinschaften und studentischen Wohnungen waren ähnliche Bett-Arrangements durchaus üblich.

18 Vgl. auch einen auf youtube zugänglichen kurzen Film vom Amsterdamer „Bed-In“: http:// www.youtube.com/watch?v=GN_ykcjHhRc; 14. Mai 2009.

19 Vgl. zu diesem Stichwort der „Unordnung“ mit Bezug sowohl auf körperliches Verhalten wie auf sprachlich-kommunikative Kontexte Scharloth (2007a). 
schine am Bett insofern ein exemplarischer Ausdruck von Unordnung, als Schreibmaschinen in ein Arbeitszimmer, zumindest aber auf einen Schreibtisch (und sicher nicht auf den Boden) gehören. Ihre Präsenz am Bett betont sowohl die Entvertikalisierung des Raumes (Schreibtischarbeit findet eben am Schreibtisch und damit ,erhöht' statt) sowie eine horizontale Entdifferenzierung von Arbeitsort und Ort der Ruhe und führt auf diese Weise zu einer Überblendung der Lebensdomänen von Arbeit und Freizeit, von Mühe und Annehmlichkeit sowie der Modalitäten Formalität und Informalität. Womit auch auf dieser kategorial abstrakteren Ebene ebenfalls wieder Unordnung produziert wird, eine Unordnung, die sich allerdings ebenso als neue Ordnung lesen lässt. Paralleles gilt für die Kleider auf dem Boden, Essen und Aschenbecher am Bett etc.

\section{Anregungskräfte}

Der Zeitgeist hat auch einen Körper. Und er scheint ihn zu brauchen. Wie an den (wenn auch nur sehr knappen) historischen Rückgriffen auf das 18. Jahrhundert gezeigt, lässt sich auch in anderen historischen Kontexten eine Verschränkung von soziokulturellen Veränderungen einerseits und Veränderungen im Habitus bzw. in der Stilistik des Körpers ${ }^{20}$ sowie in der ausdrucksbezogenen Nutzung von Raum andererseits beobachten. Der Körper erscheint dabei sowohl als Medium der Aneignung neuer kultureller ,Haltungen' und mentalitärer Dispositionen wie auch als Medium von deren Präsentation und Artikulation. ${ }^{21}$ Die transformative Potenz körperlicher Performanz scheint also in der Ausbildung, Aneignung und Diffusion kollektiver Identitäten eine zentrale Rolle zu spielen, sei dies im Kontext einer bewussten Programmatik ${ }^{22}$ oder aber als unkontrollierter Effekt der stilistischen - Anregungskraft eines bestimmten Körperauftritts.

20 Zum Stilbegriff sowie zum Verhältnis von Kollektivstil und kulturellem Stil vgl. Linke (2009).

21 Vgl. auch Fahlenbrach (2002), die die expressive Potenz von „Körpersprache“ und „Kleidersprache“ (S. 81) im Kontext der Protestbewegung der ,68er Jahre“ betont und z.B. den reduzierten, auf traditionelle Statusmarkierungen verzichtenden Kleiderstil der an den Vietnam- und Anti-Schah-Demonstrationen Teilnehmenden als Ausdruck einer gegen bürgerliche Kleiderordnungen gerichteten Verweigerungshaltung interpretiert (S. 200f.), die mit einem Verzicht auf Individualisierung einhergeht: „Der individuelle Körper wird nicht betont, sondern nurmehr, in Form von ,Massenkörpern' in Demonstrationen, Sitzblockaden usw. als politisches Argument eingesetzt" (S. 201).

22 Die Körpererziehung von Kindern ist ein Bereich der (zum Teil ganz unreflektierten) Nutzung dieser performativen Potenz. 
Mit Blick auf die Unterscheidung gesellschaftlicher Formationen bzw. Kommunikationsgemeinschaften lässt sich zudem parallel zur Sozialsemiotik von Sprache ${ }^{23}$ auch eine Sozialsemiotik des Körpers konstatieren: Sprachstile und Körperstile sind nicht unabhängig voneinander zu denken. Wie Joachim Scharloth anhand von extensiven Quellenanalysen herausgearbeitet hat, lassen sich auch innerhalb der oft als homogen dargestellten 68er-Bewegung verschiedene soziokulturelle Milieus und entsprechende Sprach- wie Körperstile erkennen. ${ }^{24}$ Die von mir hier präsentierten Raumbzw. Körpermuster wären mit Blick auf Scharloths Kategorisierung dem von ihm so genannten ,hedonistischen Selbstverwirklichungsmilieu“" zuzuordnen. Es ist dieses soziokulturelle Milieu, in welchem derjenige Sprachund Körperstil geprägt wird, der zeitgenössisch in besonders deutlichem Kontrast zum hegemonialen Habitus bürgerlicher Mittelschichten steht und entsprechend auch als besonders provokativ empfunden wird. Andererseits sind es gerade die markanten Charakteristika dieses Körperstils, die offensichtlich ein hohes Anregungspotential haben, denn sie sind, wie gezeigt, auch in anderen sozialen Formationen jenseits der Protestgeneration beobachtbar und erweisen sich in struktureller Hinsicht zudem als historisch nachhaltig. ${ }^{25}$ Und drittens lassen sich - bei allen Vorbehalten, die solchen grobrastrigen Vergleichen und Interpretationen entgegenzubringen sind - die skizzierten körperhabituellen Veränderungen auch als Abkehr von einem bürgerlichen Körperprogramm verstehen, wie es plakativ in den Kupferstichen von Daniel Chodowiecki (Abb. 2 bis 4) eingefangen ist und das spätestens seit der Mitte des 19. Jahrhunderts über die Leitformation des Bürgertums eine breite normative Wirkung entfaltet. Der in diesem Programm festgeschriebenen, schmalen und an der vertikalen Achse orientierten Leiblichkeit ${ }^{26}$ des Bürgers, welche auf ,Aufrichtigkeit ${ }^{6}$ im doppelten Sinn ${ }^{27}$ angelegt und im Gegensatz zum adligen Körperauftritt demonstrativ nicht raumgreifend konzipiert ist, wird im Körperprogramm der 68er Jahre nun ein auf neue Weise raumgreifender Habitus entgegengesetzt, der die Vertikalität aufweicht und im Gegensatz zum klaren Körperprogramm

23 Vgl. hierzu für die ,Sprache der 68er einschlägig Mattheier (2001), Scharloth (2007a) und (2007b) sowie Scharloth (2011).

24 Scharloth (2011).

25 Die Kombination von von Arbeits- und Schlafzimmer sowie die räumliche Verbindung von Kochen und Wohnen (auch ohne den Sachzwang einer kleinen Wohnung) sind seit den 70er Jahren zunehmend zum Standard mittelständischen Wohnens geworden, nicht zuletzt in intellektuellen Kreisen.

26 Dieses körperliche Leitbild kommt am deutlichsten im Herrenkostüm bzw. im Körperauftritt des Mannes zum Ausdruck, vgl. etwa auch Brändli (1998).

27 Vgl. Warneken (1990). 
der Chodowiecki'schen Tafeln die Akteure gerade nicht auf ein einheitliches Haltungsmuster verpflichtet. ${ }^{28}$

Damit lassen sich, wie bereits angedeutet, die hier vorgelegten Beobachtungen und Interpretationen unter den bereits oben eingeführten Begriff der Entdifferenzierung stellen, mit dem Jürgen Link - unter Rückgriff auf sein Konzept des Normalismus - die Effekte des von ihm diagnostizierten „anti-normalistischen Impetus “ ${ }^{\text {‘29 }}$ zusammenfasst, welchen er für die heute unter dem Label „1968“ subsummierten sozialen Bewegungen als konstitutiv erachtet. In einem Beitrag zur „Spezifizität der, Bewegung von Achtundsechzig““ (Link 2001) ortet Link diese „Ent- bzw. Umdifferenzierung ${ }^{\text {‘630 }}$ vor allem auf der Makroebene gesellschaftlicher Handlungsfelder und sozialer bzw. kultureller Kategorisierungen. Beispiele sind etwa die In-Frage-Stellung und Aufweichung der Disziplinenbildung in den Wissenschaften, die kritische Auseinandersetzung mit der Ausgrenzung von Behinderten oder Psychiatrisierten sowie mit den „Normalitätsgrenzen“"31 zwischen Kunst und Leben oder Kunst und Politik. Entdifferenzierung wird damit zu einer möglichen Form der Aushebelung bzw. der Infragestellung von Normalismus. $^{32}$

Eben dieses strukturelle Grundmuster der Ent- bzw. Umdifferenzierung lässt sich nun aber - wie gezeigt - auch auf der Mikroebene der Materialität alltäglicher Raum-Leiblichkeit identifizieren, sei dies in der alltagsweltlichen Wohn-Topographie, wo die etablierten Grenzen zwischen Arbeits-, Schlaf-, Ess- und Wohnraum aufgehoben werden und Insignien dieser Funktionsräume - Schreibmaschine, Staubsauger, abgelegte Kleider - in neuer Vermischung zusammenrücken, oder in der Entvertikalisierung des Raum-Körper-Bezugs, die mit dem neuen Sitzen ebenso wie mit dem aktiven Liegen verbunden ist und die symbolische Macht-Konnotiertheit von Vertikalität im Abbau dieser Achse sowohl demonstrativ ausstellt als auch demontiert.

Die Alltagsperformanz von Körperauftritt und Raumnutzung erscheint damit als Medium des Ausdrucks wie der Aneignung eines neuen Weltund Selbstverständnisses, das in dieser Materialisierung sowohl eine ebenso handfest-konkrete wie symbolisch-metaphorische Fassung findet. Und es

28 Mit dieser Neuformierung von Körperauftritt und Raumbezug wendet sich die ,68er Generation" nun allerdings nicht gegen eine als genuin ,anders“ konstruierte Konkurrenzformation, wie sie der Adel für das Bürgertum des 18. Jahrhunderts darstellte, sondern gegen die soziale und kulturelle Formation, der sich die Mehrheit der Protestbewegung zumindest der Herkunft nach selbst zurechnet.

29 Link (2001, S. 71).

30 Ebd.

31 Ebd.

32 Link (2001, S. 72). 
ist diese materielle Ebene, diese Körperlichkeit des Protests, die das Anregungspotenzial entwickeln kann, das zur interaktiven Verbreitung einer ,Haltung und ,Einstellung'von Körper zu Körper beiträgt, die jenseits (und allenfalls auch vor) jeder bewussten und abstrakteren Reflexion der gesellschaftlichen Umbrüche zur Ausbildung eines bestimmten Generationengefühls der 68er beigetragen hat.

\section{Literatur}

Brändli, Sabina (1998): „Der herrlich biedere Mann“. Vom Siegeszug des bürgerlichen Herrenanzuges im 19. Jahrhundert. Zürich.

Breuer, Gerda/Peters, Andrea/Plüm, Kerstin (Hg.) (1999): Die 60er. Positionen des Designs. Köln/Wuppertal.

Cassirer, Ernst (1985): Mythischer, ästhetischer und theoretischer Raum. In: Cassirer, Ernst: Symbol, Technik, Sprache. Hg. von Ernst Wolfgang Orth und Michael Krois. Hamburg, S. 93-119.

Fahlenbrach, Kathrin (2002): Protestinszenierungen. Visuelle Kommunikation und kollektive Identitäten in Neuen Sozialen Bewegungen. Wiesbaden.

Grob, Marion (1985): Das Kleidungsverhalten jugendlicher Protestgruppen in Deutschland im 20. Jahrhundert am Beispiel des Wandervogels und der Studentenbewegung. Münster.

Haller, Joachim (1970): Der gute Ton im Umgang mit Menschen. Mit 70 Zeichnungen im Text und zahlreichen Fotos auf 16 Kunstdrucktafeln. 8. Aufl. München.

Klemm, Barbara (1999): Unsere Jahre. Bilder aus Deutschland 1968-1998. München.

Lichtenberg, Georg Christoph (1971): Handlungen des Lebens. Erklärungen zu 72 Monatskupfern von Daniel Chodowiecki. Neudruck. Stuttgart.

Link, Jürgen (2001): Intensität, Entdifferenzierung, Kulturrevolution und Normalismus. Zur Spezifizität der „Bewegung von Achtundsechzig“. In: Ott, Ulrich/Luckscheiter, Roman (Hg.): Belles lettres/Graffiti. Soziale Phantasien und Ausdrucksformen der Achtundsechziger. Göttingen, S. 69-78.

Linke, Angelika (1996): Sprachkultur und Bürgertum. Zur Mentalitätsgeschichte des 19. Jahrhunderts. Stuttgart.

Linke, Angelika (2009): Stil und Kultur. In: Fix, Ulla/Gardt, Andreas/Knape, Joachim (Hg.): Rhetorik und Stilistik. Ein internationales Handbuch historischer und systematischer Forschung. Zweiter Teilb. Berlin/New York, S. 1131-1144.

Linke, Angelika (2010): Historische Semiotik des Leibes in der Kommunikation. Zur Dynamisierung von Körper und Sprache im ausgehenden 17. und im 18. Jahrhundert. In: Deppermann, Arnulf/Linke, Angelika (Hg.): Sprache intermedial. Stimme und Schrift, Bild und Ton. (=Jahrbuch des Instituts für Deutsche Sprache 2009). Berlin/New York, S. 129-162. 
Linke, Angelika/Scharloth, Joachim (Hg.) (2008): Der Zürcher Sommer 1968. Zwischen Krawall, Utopie und Bürgersinn. Zürich.

Mattheier, Klaus J. (2001): Protestsprache und Politjargon. Über die problematische Identität einer ,Sprache der Achtundsechziger'. In: Ott, Ulrich/Luckscheiter, Roman (Hg.): Belles lettres/Graffiti. Soziale Phantasien und Ausdrucksformen der Achtundsechziger. Göttingen, S. 79-90.

Ruetz, Michael (1997): 1968 - Ein Zeitalter wird besichtigt. 323 Photographien mit Texten von Sachsse, Rolf/Broder, Henryk M./Ruetz, Michael. Frankfurt a.M.

Sachsse, Rolf (1997): Geschichte Erinnern - Eine Gebrauchsanweisung. In: Ruetz, Michael: 1968 - Ein Zeitalter wird besichtigt. 323 Photographien mit Texten von Sachsse, Rolf/Broder, Henryk M./Ruetz, Michael. Frankfurt a.M., S. 10-21.

Scharloth, Joachim (2005): Sprachnormen und Mentalitäten. Sprachbewusstseinsgeschichte in Deutschland im Zeitraum von 1766 und 1785. (= RGL 255). Tübingen.

Scharloth, Joachim (2007a): 1968 und die Unordnung in der Sprache. Kommunikationsstrukturelle und sozialstilistische Untersuchungen. In: Pappert, Steffen (Hg.): Die (Un)Ordnung des Diskurses. Leipzig, S. 11-36.

Scharloth, Joachim (2007b): Die Sprache der Revolte. Linke Wörter und avantgardistische Kommunikationsstile. In: Klimke, Martin/Scharloth, Joachim (Hg.): 1968. Handbuch zur Kultur- und Mediengeschichte der Studentenbewegung. Stuttgart, S. 223-234.

Scharloth, Joachim, (2011): 1968. Eine Kommunikationsgeschichte. Paderborn.

Schittenhelm, Rosemarie (1967): Man wird dich lieber haben. Anstandsbuch für junge Damen. 5., völlig neu bearbeitete und erweiterte Aufl. Stuttgart.

Warneken, Bernd Jürgen (1990): Biegsame Hofkunst und aufrechter Gang. Körpersprache und bürgerliche Emanzipation um 1800. In: Warneken, Bernd Jürgen (Hg.) (1990): Der aufrechte Gang. Zur Symbolik einer Körperhaltung. Tübingen, S. 11-23.

Wirth, Uwe (2008): Vorüberlegungen zu einer Logik der Kulturforschung. In: Wirth, Uwe (Hg.): Kulturwissenschaft. Eine Auswahl grundlegender Texte. Frankfurt a.M., S. 9-67. 